Article

\title{
Partial Discharge Measurements in a High Voltage Gas Insulated Transmission Line Insulated with $\mathrm{CO}_{2}$
}

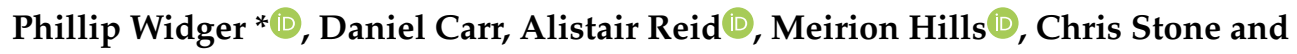
A. (Manu) Haddad $(\mathbb{D}$

School of Engineering, Cardiff University, The Parade, Cardiff CF24 3AA, UK; CarrD3@cardiff.ac.uk (D.C.); ReidA3@cardiff.ac.uk (A.R.); HillsMT@cardiff.ac.uk (M.H.); StoneCT1@cardiff.ac.uk (C.S.);

Haddad@cardiff.ac.uk (A.H.)

* Correspondence: widgerp@cardiff.ac.uk

Received: 7 May 2020; Accepted: 3 June 2020; Published: 5 June 2020

\begin{abstract}
This paper uses practical experimentation to analyse the effect of replacing $\mathrm{SF}_{6}$ with pure $\mathrm{CO}_{2}$ in conventional gas insulated transmission line sections by studying partial discharge measurements taken with applied voltages up to $242 \mathrm{kV}$ (rms). The results can also help in understanding the properties of new alternative gas mixtures which can be utilised with a ratio of up to and over $95 \%$ $\mathrm{CO}_{2}$. The experiments undertaken involved filling a gas insulated line demonstrator with 3 bars of $\mathrm{CO}_{2}$ and applying voltages up to $242 \mathrm{kV}$ in both clean conditions and particle-contaminated enclosure conditions. The results demonstrate that $\mathrm{CO}_{2}$ can be used to insulate gas equipment without breakdown at high voltage, however, a higher gas-filling pressure may be needed to reduce the partial discharge found in the tests presented in this paper. Another aspect of the work showed that partial discharge (PD) measurements from internal ultra-high frequency (UHF) sensors compared with a direct measurement from a capacitive divider both clearly showed the effect of contaminating particles in $\mathrm{CO}_{2}$. However, the PD divider measurements also showed considerable external PD on the outside of the gas compartment, leading to the conclusion that UHF sensors are still regarded as having the highest sensitivity and noise immunity for gas insulated switchgear (GIS) or gas insulated transmission line (GIL) systems including when the equipment is insulated with $\mathrm{CO}_{2}$.
\end{abstract}

Keywords: gas insulated transmission lines (GILs); partial discharge (PD); sulphur hexafluoride $\left(\mathrm{SF}_{6}\right)$, carbon dioxide $\left(\mathrm{CO}_{2}\right)$; ultra high frequency (UHF) sensors

\section{Introduction}

The use of gas insulated busbars (GIB) and transmission lines (GIL) is common practice within the high voltage power industry when insulated with compressed sulphur hexafluoride $\left(\mathrm{SF}_{6}\right)$ gas to enable compact electrical substations and transmit large amounts of energy across large distances or in urban locations or hard-to-reach areas such as underground river crossings that overhead power lines may not be able to facilitate. The use of $\mathrm{SF}_{6}$ is increasing in the UK [1] and is facing growing scrutiny considering that it is a global warming gas with an estimated global warming potential of 23,500 times that of carbon dioxide $\left(\mathrm{CO}_{2}\right)$ and an atmospheric lifetime of 3200 years, making it one of the most detrimental global warming gases known to humankind when released into the atmosphere [2,3]. Current research efforts are focusing on the replacement of $\mathrm{SF}_{6}$ with alternatives such as $\mathrm{C}_{4} \mathrm{~F}_{7} \mathrm{~N}[4,5], \mathrm{C}_{5} \mathrm{~F}_{10} \mathrm{O}[6,7]$ and $\mathrm{CF}_{3} \mathrm{I}[8,9]$ in relatively small mixture quantities $(1-30 \%)$ as a partial pressure gas mixture with large quantities of $\mathrm{CO}_{2}$ (up to $95 \%$ ) as a buffer gas making up a component gas mixture of two or three gases. It is, therefore, important that the partial discharge characteristics within high voltage gas insulated transmission lines (GIL), busbars and gas insulated switchgear (GIS) are studied in order to ensure accurate diagnostic measurement useful for the replacement of $\mathrm{SF}_{6}$ 
with a component gas mixture largely consisting of $\mathrm{CO}_{2}$ or pure $\mathrm{CO}_{2}$. Additional beneficial research, connected to the trials of $\mathrm{CO}_{2}$ as an insulation medium, in small vessels to understand streamer and breakdown characteristics has been conducted by [10] and the partial discharge (PD) inception and breakdown voltages studied at high pressure of a model spacer in [11]. This paper examines the use of non-conventional PD measurements directly coupled using a capacitive divider connected to the studied GIL and compared to conventional ultra-high frequency (UHF) sensors.

Partial discharge is primarily used as an indication of equipment degradation, detecting the state of insulation performance of the system as a whole and a method, in this research, of assessing the use of $\mathrm{CO}_{2}$ as a direct replacement for $\mathrm{SF}_{6}$ in practical GIB and GIL. It is also useful to note that studying the effects of pure $\mathrm{CO}_{2}$ is beneficial considering that the alternative mixtures have gases that degrade over time, leading to a change in mixture ratio during an equipment's lifetime, or in the case of fault conditions, where some arcing occurs or continuous partial discharge affects the gas ratio [12]. The use of $\mathrm{CO}_{2}$ in gas insulated equipment on high voltage networks, if highly widespread in the future, could prove to be a sustainable way to offset carbon emissions and use waste production of $\mathrm{CO}_{2}$ from other processes in order to capture and usefully store otherwise emitted global warming gases.

\section{Practical Test Arrangement and Methods}

During the practical experimentation undertaken in this paper, full-scale gas insulated equipment, designed to be utilised with $\mathrm{SF}_{6}$ as an insulation medium at a rated voltage of $420 \mathrm{kV}$ on the UK network, was used to build the demonstrator shown in Figure 1 in the high voltage laboratory at Cardiff University. The normal operating conditions of this equipment when insulated with a minimum filling pressure of 2.9 bar of $\mathrm{SF}_{6}$ are shown in Table 1 . The voltage ratings shown in Table 1 are consistent with IEC 62771-203 equipment ratings for high voltage gas insulated equipment [13].

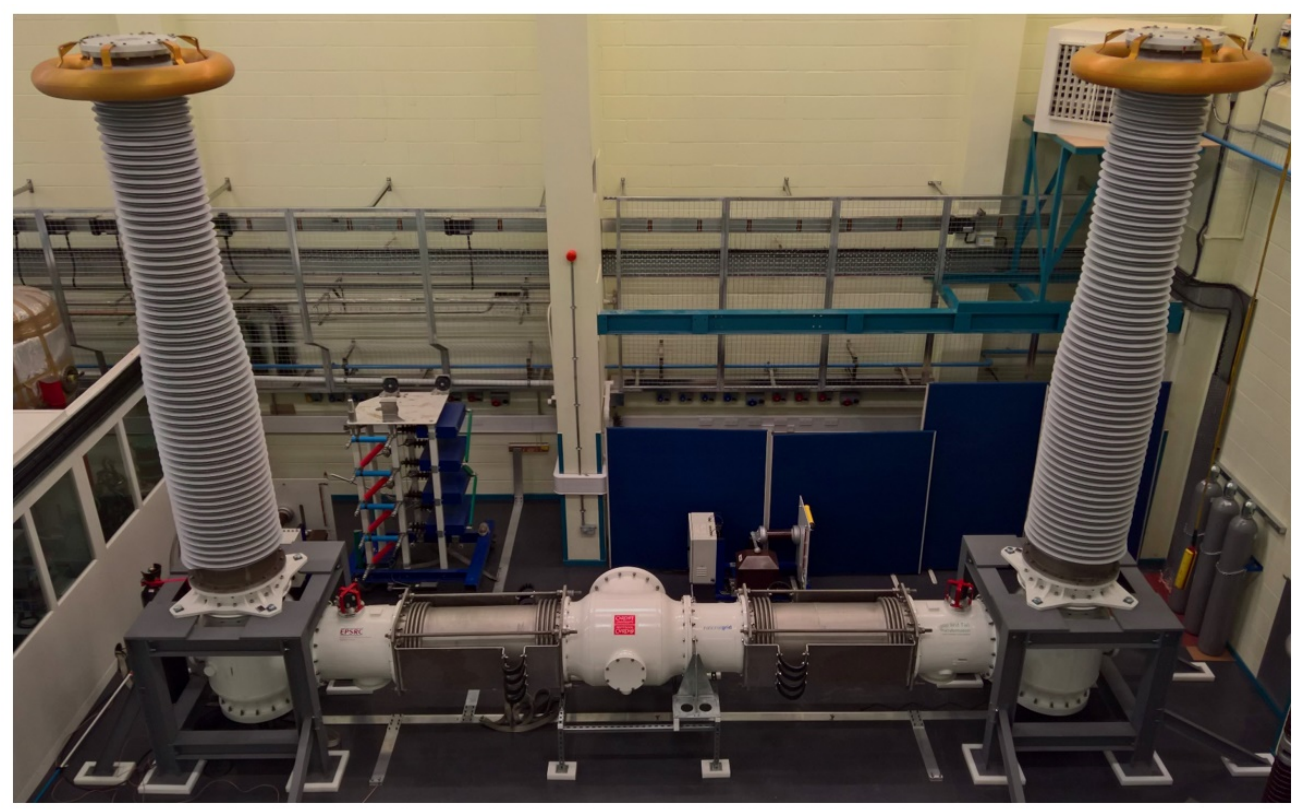

Figure 1. Gas insulated line demonstrator constructed and installed at Cardiff University.

The gas insulated line demonstrator was constructed of the sections shown in Figure 2, with connecting elbow joints either end allowing the use of two high voltage bushings. The central gas zone consisted of a $4.915 \mathrm{~m}$ central conductor suspended between two epoxy resin cone spacers which seal the gas zone at both ends. This central section of the gas insulated line demonstrator also contains two UHF capacitive plate sensors at both ends of the GIL section and is equipped with a gas-filling port. The outer enclosure length of this section is $5.275 \mathrm{~m}$ in length. The dimensions of the outer conductor and inner enclosure of the constructed gas insulated line demonstrator are shown in Figure 3. 
Table 1. Specifications of the gas insulated demonstrator at Cardiff when filled with pure $\mathrm{SF}_{6}$ [13].

\begin{tabular}{ccc}
\hline Specification & Abbreviation & IEC 62271-203 (2003) Equipment Rating \\
\hline Rated voltage/rated frequency & $\mathrm{Ur} / \mathrm{fr}$ & $420 \mathrm{kV} / 50 \mathrm{~Hz}$ \\
Power frequency withstand voltage & $\mathrm{Ud}$ & $650 \mathrm{kV}$ \\
Lightning impulse withstand voltage & $\mathrm{Up}$ & $1425 \mathrm{kV}$ \\
Switching impulse withstand voltage & $\mathrm{Us}$ & $1050 \mathrm{kV}$ \\
Rated auxiliary voltage & $\mathrm{Ua}$ & $125 \mathrm{~V} \mathrm{~d} . \mathrm{c}$. \\
Nominal current-busbars and bays & $\mathrm{Ir}$ & $4000 \mathrm{~A}$ \\
Short-time withstand current/duration & $\mathrm{Ik} / \mathrm{tk}$ & $63 \mathrm{kA} / 1 \mathrm{~s}$ \\
Peak withstand current & $\mathrm{Ip}$ & $157 \mathrm{kA}$ \\
$\mathrm{CB} \mathrm{SF}_{6}$ gas pressure: filling/alarm/minimum & Pre/Pae/Pme & $7.5 / 6.8 / 6.5 \mathrm{bar}$ \\
(at 20 ${ }^{\circ} \mathrm{C}$ ) & & $3.5 / 3.1 / 2.9 \mathrm{bar}$ \\
$\mathrm{GIS} \mathrm{SF}_{6}$ gas pressure: filling/alarm/minimum & Pre/Pae/Pme & \\
(at $20^{\circ} \mathrm{C}$ ) &
\end{tabular}

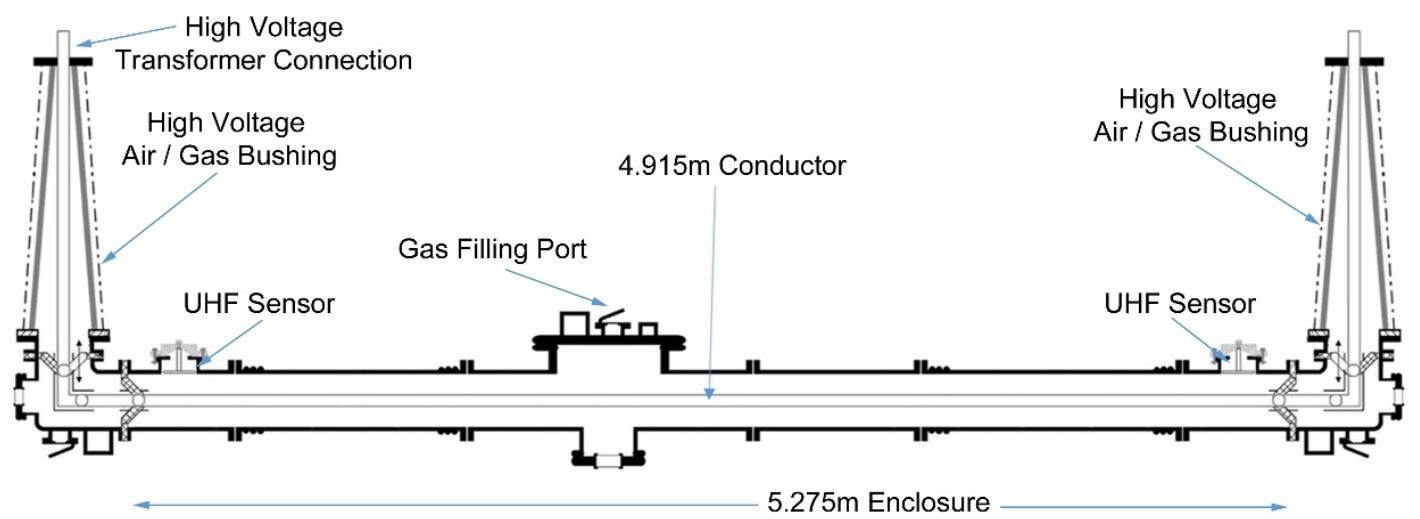

Figure 2. Gas insulated line demonstrator sections and dimensions.

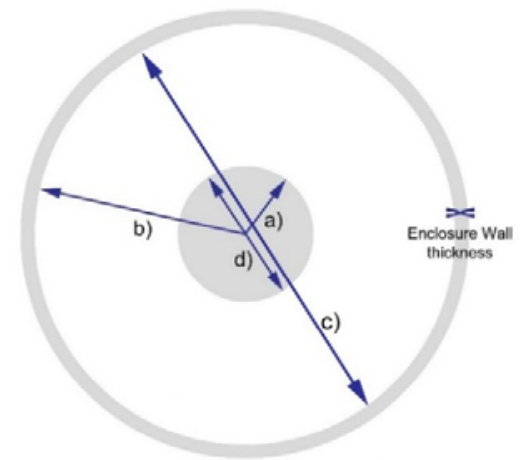

a) Outer conductor radius $=95.5 \mathrm{~mm}$

b) Inner enclosure radius $=270 \mathrm{~mm}$

c) Inner enclosure diameter $=540 \mathrm{~mm}$

d) Outer conductor diameter $=191 \mathrm{~mm}$

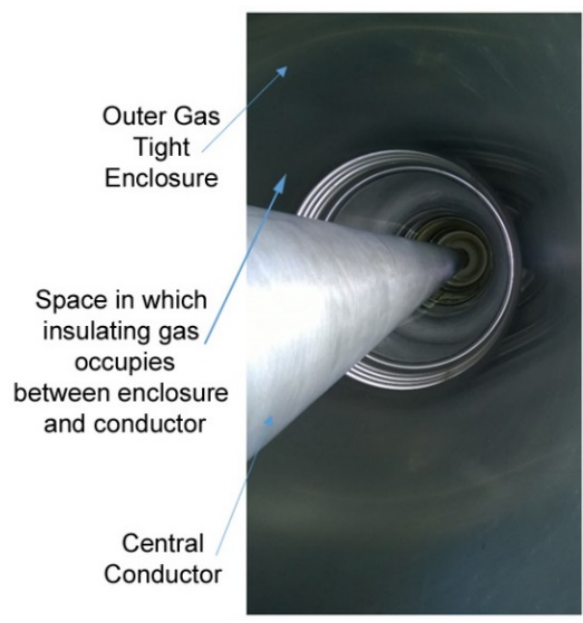

Figure 3. Gas insulated line inner conductor and outer enclosure dimensions.

Considering that the gas insulated line demonstrator has an outer conductor diameter of $191 \mathrm{~mm}$ and an inner enclosure diameter of $540 \mathrm{~mm}$ it can be calculated that the outer conductor radius $\left(R_{a}\right)$ is $95.5 \mathrm{~mm}$ and the inner enclosure radius $\left(R_{b}\right)$ is $270 \mathrm{~mm}$. Using the dimensions shown in Figure 3 , it can be verified that the dimensions are close to the desired theoretical values, as is normally considered when designing gas insulated equipment in order to create a near-uniform electric field distribution which helps the insulation performance of the insulating gas under applied voltage conditions:

$$
\ln \left(\frac{R_{b}}{R_{a}}\right)=\ln \left(\frac{270}{95.5}\right)=1.039
$$




$$
\ln \left(\frac{R_{b}}{R_{a}}\right)=1 \quad \text { is the ideal }
$$

Considering the IEC/BS EN standard 62771-203 [13] and 62271-204 [14], which recommend the same method for partial discharge detection for both gas insulated transmission lines and switchgear, the test voltages shown in Table 2 were used for practical testing to show system rating voltage responses. To conform with the standards, a pre-stress voltage would normally be applied before a PD measurement is taken. However, in these experiments, this was not possible due to the available transformer rating limitations. For a system with a solidly earthed neutral, the applied test voltage for partial discharge measurement $\left(\mathrm{U}_{\text {pd-test }}\right)$ to a single-phase enclosure design, such as the gas insulated line demonstrator used in this work, is calculated using the following equation [13] and applied for any time greater than $1 \mathrm{~min}$ :

$$
U_{p d-t e s t}=1.2 U_{r} / \sqrt{3}
$$

Table 2. Gas insulated line demonstrator partial discharge test voltage levels.

\begin{tabular}{ccc}
\hline $\begin{array}{c}\mathbf{U}_{\mathrm{r}} \\
\begin{array}{c}\text { Gas Insulated Line Demonstrator } \\
\text { Rated Voltage }\end{array}\end{array}$ & $\begin{array}{c}\mathbf{U}_{\text {ph-ea }} \\
\text { Nominal } \\
\text { System Phase to earth } \\
\text { Voltage }\end{array}$ & $\begin{array}{c}\mathbf{U}_{\text {pd-test }} \\
\text { Test Voltage for PD Measurement } \\
\mathbf{U}_{\text {ph-test }}(>\mathbf{~} \text { min) }\end{array}$ \\
\hline $300 \mathrm{kV}$ & $173 \mathrm{kV}$ & $208 \mathrm{kV}$ \\
$362 \mathrm{kV}$ & $209 \mathrm{kV}$ & $251 \mathrm{kV}$ \\
$400 \mathrm{kV}$ & $231 \mathrm{kV}$ & $277 \mathrm{kV}$ \\
$420 \mathrm{kV}$ & $242 \mathrm{kV}^{1}$ & $291 \mathrm{kV}$ \\
\hline
\end{tabular}

${ }^{1}$ Maximum applied voltage achieved during practical testing was $242 \mathrm{kV}$ due to external PD.

It is also important to note that, as described in $[13,14]$, the maximum permissible partial discharge level is $5 \mathrm{pC}$ at the voltages specified in Table 2 . However, in these experiments, an oil-filled transformer was used to apply the voltage, which means that the system as a whole may be considered acceptable if the discharge level does not exceed $10 \mathrm{pC}$, as the PD of the transformer is included in the measured result.

Using the IEC/BS EN 60270 for high voltage partial discharge test techniques [15], the upper $\left(f_{1}\right)$ and lower frequency $\left(f_{2}\right)$ limits of the wide band partial discharge equipment (coupled to the high voltage capacitive divider capacitors or power seperation filter), as determined suitable for high voltage equipment, can be set as follows:

$$
\begin{aligned}
30 \mathrm{kHz} & \leq f_{1} \leq 100 \mathrm{kHz} \\
f_{2} & \leq 1 \mathrm{MHz} \\
100 \mathrm{kHz} & \leq f_{2} \leq 900 \mathrm{kHz}
\end{aligned}
$$

For the following practical tests, the measurement system (Omicron MPD 540) shown in Figure 4a was set to the following values:

$$
\begin{aligned}
\mathrm{f}_{1}=30 \mathrm{kHz}, \mathrm{f}_{2} & =130 \mathrm{kHz} \\
\text { Frequency integration } & =80 \mathrm{kHz} \pm 50 \mathrm{kHz} .
\end{aligned}
$$

These values were set using a trial and error method which identifies the frequency range that can be used without including specific noise or outside interference detected by the system in and around the laboratory.

Figure $4 \mathrm{~b}$ shows the signal conditioning used to couple the UHF sensors to the PD measurement system (Omicron UHF 620 and MPD 600). While the bandwidth of the UHF sensor is in the range of $2 \mathrm{GHz}$, measurements were converted to a lower frequency range for compatibility with the phase 
resolved analysis system (the results of which are shown in Section 3). The recorded frequency range of this apparatus was as follows:

$$
\mathrm{f}_{1}=399.3 \mathrm{MHz}, \mathrm{f}_{2}=400.8 \mathrm{MHz},
$$

Frequency integration $=400 \mathrm{MHz} \pm 750 \mathrm{kHz}$.

The arrangement of all equipment used to carry out the practical tests undertaken in this paper are shown in Figure 5. The gas insulated demonstrator was filled with 3 bar of $\mathrm{CO}_{2}$, with a technical specification of $99.8 \% \mathrm{CO}_{2}$ [16]. The gas insulated demonstrator was tested as both a clean system and as a particle-filled system with $10 \times 1 \mathrm{~mm}$ diameter stainless steel spheres placed as free floating particles within the central gas insulated enclosure [17].

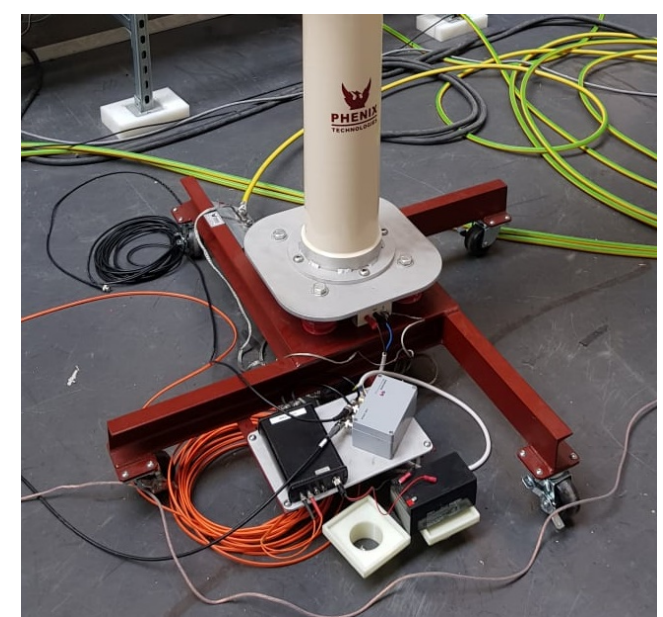

(a)

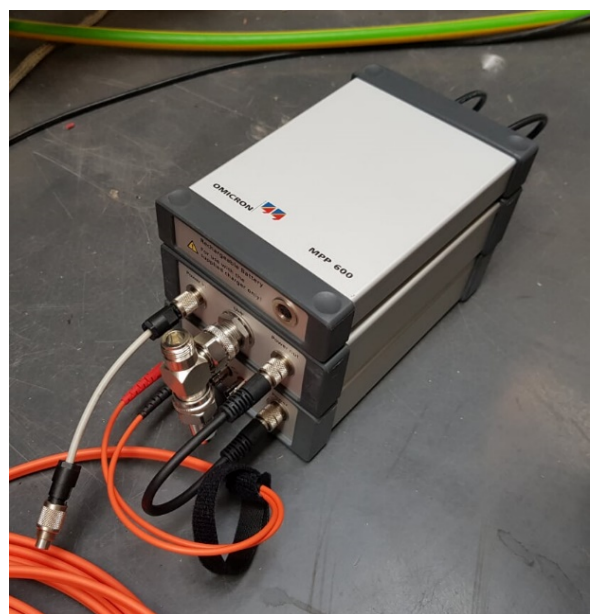

(b)

Figure 4. (a) Optically isolated partial discharge (PD) equipment coupled to a coupling capacitor of $1 \mathrm{nF}$ or power seperation filter; (b) ultra high frequency (UHF) capacitive sensor coupled with optically isolated PD measurement equipment.

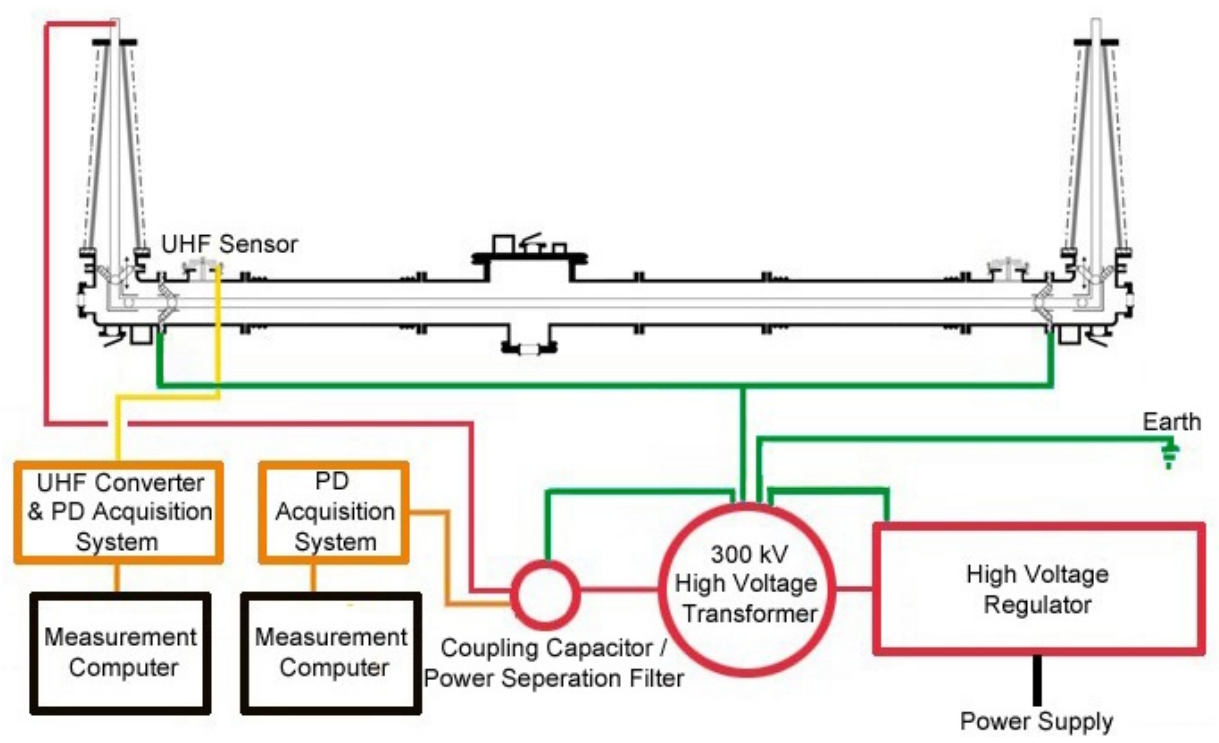

Figure 5. Practical test arrangement including gas insulated line demonstrator, partial discharge acquisition equipment, measuring computers and high voltage transformer. 


\section{Results}

This section gives a description of the experimental results for both the clean gas insulated line demonstrator filled with pure $\mathrm{CO}_{2}$ and a system with artificial particle contamination to show their effects on $\mathrm{PD}$ measurements and $\mathrm{CO}_{2}$ insulation.

\subsection{Partial Discharge Measurement-Clean System}

The first experimental tests undertaken using the gas insulated line demonstrator, filled with 3 bars of $\mathrm{CO}_{2}$, are shown in Figure 6 at applied AC voltages of $50 \mathrm{kV}, 100 \mathrm{kV}, 150 \mathrm{kV}$ and $200 \mathrm{kV}$ (rms). The PD measurements shown in Figure 6 are representative of the full system including the AC oil-filled transformer, the $\mathrm{CO}_{2}$ gas-filled bushings and the central section of the gas insulated line when measured using the coupling capacitor directly connected to the AC voltage transformer. The results indicate that the system as a whole has a peak apparent charge of approximately $10 \mathrm{pC}$ but as the voltage was increased, a PD level up to $100 \mathrm{pC}$ can be seen. It was found that the largest PD signals, occurring at approximately the 3rd quadrant of the AC waveform, were largely a result of external PD around the outside of one of the air bushings at the end of the gas insulated line demonstrator. The end of the bushing was approximately $1.5 \mathrm{~m}$ away from the external wall of the lab and hence the PD generated could not be reduced. This external PD was found to occur at the connection point of an air bushing and was located using a UV camera that is capable of detecting corona in daylight. The rest of the AC cycle shows lower PD levels averaging less than or approximately $10 \mathrm{pC}$, even though this gas insulated equipment is filled with $\mathrm{CO}_{2}$ instead of $\mathrm{SF}_{6}$ which has a much higher dielectric strength. It also shows that, even though the equipment is filled to 3 bar of $\mathrm{CO}_{2}$, which is 0.5 bar below the normal filling pressure of this equipment when filled with $\mathrm{SF}_{6}$ and close to the minimum allowed pressure of the equipment when in service, this pressure of $\mathrm{CO}_{2}$ is still able to insulate the equipment up to $200 \mathrm{kV}$ rms.

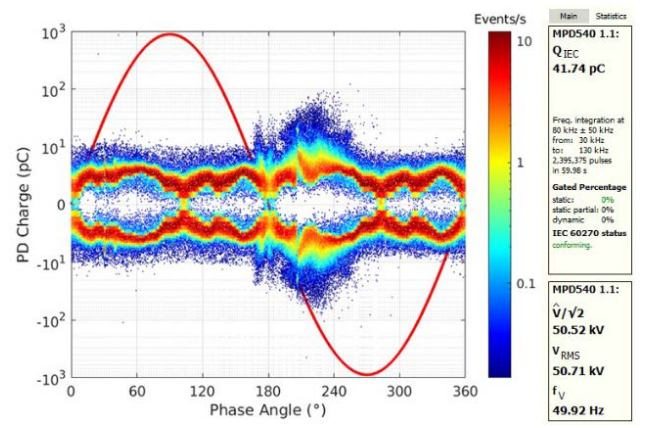

(a) Applied voltage: $50 \mathrm{kV} \mathrm{rms}$

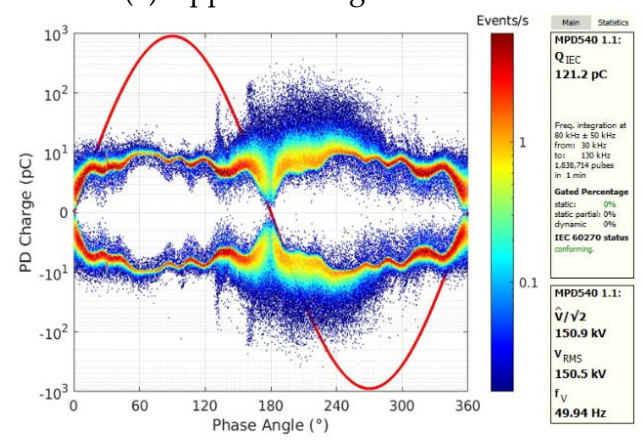

(c) Applied voltage: $150 \mathrm{kV} \mathrm{rms}$

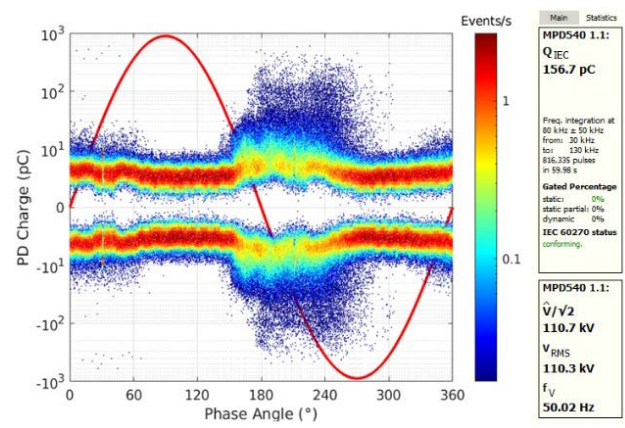

(b) Applied voltage: $100 \mathrm{kV} \mathrm{rms}$

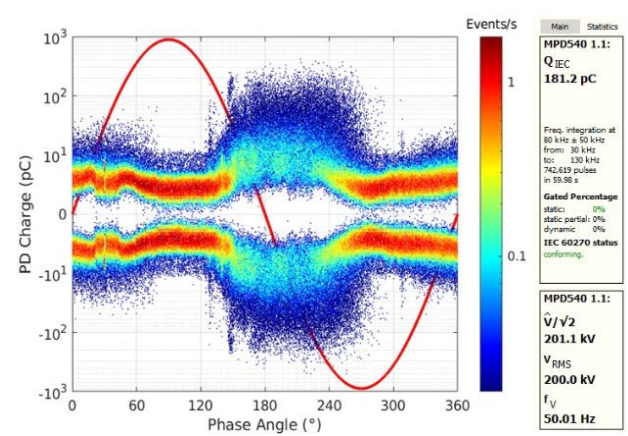

(d) Applied voltage: $200 \mathrm{kV} \mathrm{rms}$

Figure 6. PD measurement of a gas insulated line demonstrator filled with 3 bar $\mathrm{CO}_{2}$-clean system-measured directly with the high voltage divider coupling capacitor at applied rms voltages of (a) $50 \mathrm{kV}$, (b) $100 \mathrm{kV}$, (c) $150 \mathrm{kV}$, and (d) $200 \mathrm{kV}$. Close investigation of the PD measurement indicated that high activity regions in the 1st and 3rd quadrants were found to be external discharges around the connecting air bushings. 
PD measurements were also undertaken for the gas insulated line demonstrator filled with 3 bar $\mathrm{CO}_{2}$ at the phase-to-earth system voltages and the calculated PD measurement voltage shown in Table 2. The maximum permissible voltage applied to the demonstrator was $242 \mathrm{kV}$ due to external PD on the outside of the air insulated bushing. This meant that the tests, shown in Figure 7, were able to demonstrate the performance of the clean system at a rated line voltage of $300 \mathrm{kV} \mathrm{rms}$ using the phase-to-earth voltage of $173 \mathrm{kV}$ rms and the PD measurement voltage of $208 \mathrm{kV}$ rms. For the other rms voltage ratings of 362, 400 and $420 \mathrm{kV}$, the tests undertaken demonstrated the performance of the clean gas insulated line at the phase-to-earth voltage ratings only of 209, 231 and $242 \mathrm{kV}$, respectively, as shown in Figure 7, and could not be performed at the PD measurement voltage level. The results show that the gas insulated line filled with $\mathrm{CO}_{2}$ at 3 bar can insulate the conductor to the enclosure gas gap distance without a breakdown of the gas at $242 \mathrm{kV}$ rms. However, there is still significant and increasing external PD from the outside of the air bushing centered at approximately $200^{\circ}$ of the AC waveform. The results also seem to show that additional PD artefacts appear outside the $125-285^{\circ}$ window, with a potential area of partial discharge activity located approximately around $300^{\circ}$ from $208 \mathrm{kV}$ onwards. This PD activity is indicative of external PD such as corona or surface discharge, it could be across the surface of the cone insulating spacer, but is likely to be a continuation of the external PD seen at lower voltages.

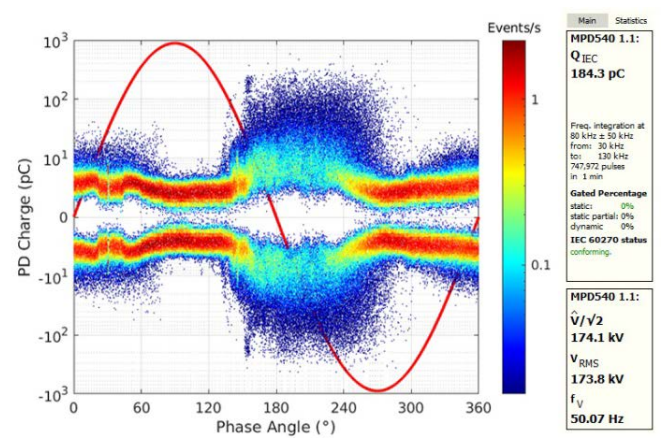

(a) Applied voltage: $173 \mathrm{kV} \mathrm{rms}$

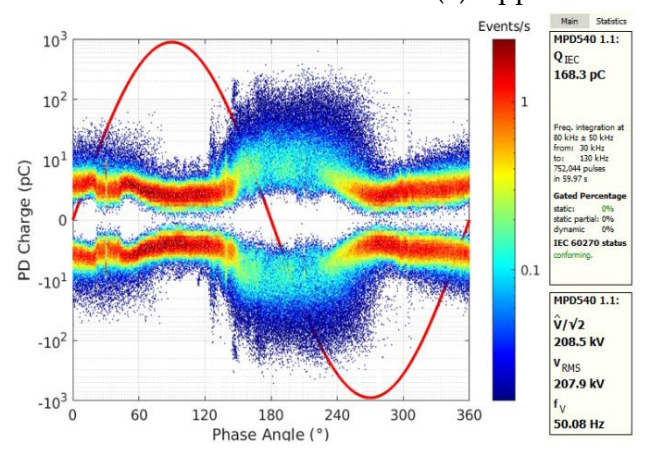

(b) Applied voltage: $208 \mathrm{kV} \mathrm{rms}$

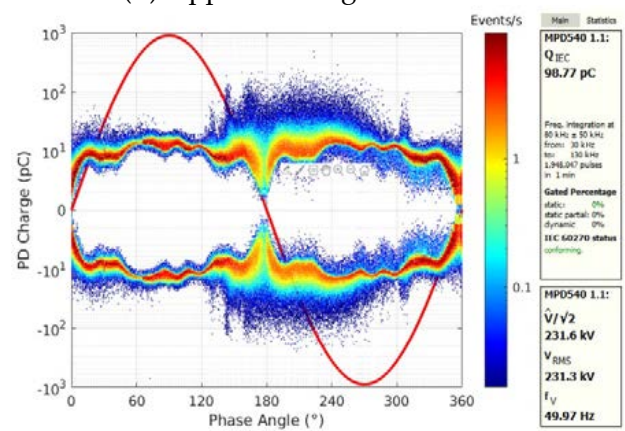

(d) Applied voltage: $231 \mathrm{kV} \mathrm{rms}$

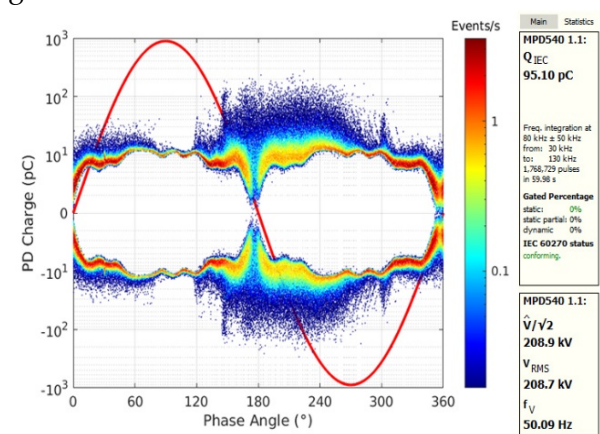

(c) Applied voltage: $209 \mathrm{kV} \mathrm{rms}$

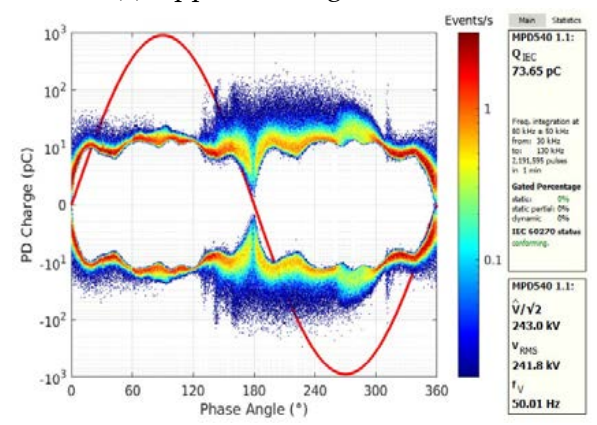

(e) Applied voltage: $242 \mathrm{kV}$ rms

Figure 7. PD measurement of a gas insulated line demonstrator filled with 3 bar $\mathrm{CO}_{2}$-clean system-measured directly with a HV coupling capacitor for shown system rms voltages. 


\subsection{Partial Discharge Measurement-Particle-Contaminated System}

In the next set of tests, ten $\times 1 \mathrm{~mm}$ diameter stainless steel spheres [17] were placed inside along the whole length of the central enclosure of the gas insulated line demonstrator in order to simulate the effect of metal particles and the type of PD pattern these may cause in a gas insulated line filled with $\mathrm{CO}_{2}$ instead of $\mathrm{SF}_{6}$. Figure 8 shows the PD results for applied rms voltages of 50,75 and $100 \mathrm{kV}$ to the system with the presence of particle contamination. The PD measurements of the particle-contaminated gas insulated line at 3 bar $\mathrm{CO}_{2}$ were achieved using the high voltage arm of a divider as the coupling capacitor. The effect of the particles on the system, shown in Figure 8a, can be compared to the clean system in Figure 6a, and there was a negligible amount of PD on the rising half of the positive AC cycle but otherwise little difference. In Figure 8b, the system contaminated with particles at $75 \mathrm{kV}$ is shown, and considerable PD was detected on the rising half of the positive AC cycle. In Figure 8c, the voltage applied to the contaminated gas insulated line was increased to $100 \mathrm{kV}$, and it can be observed that there is significant PD in the measured PD frequency range on both rising edges of the positive and negative cycles of the AC waveform. It is likely that the measurements appear as clusters at certain orders of magnitude because of a capacitive effect, likely due to the particles bouncing on the inside of the enclosure of the gas insulated line section but not as free-floating particles, as explained in the next section on UHF measurements.

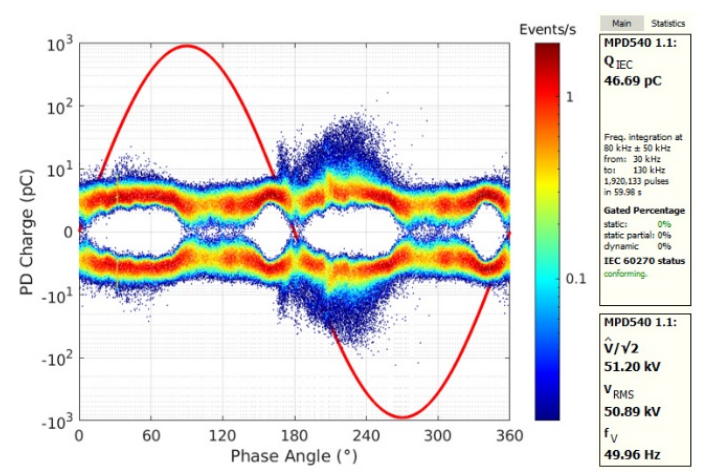

(a) Applied voltage: $50 \mathrm{kV} \mathrm{rms}$

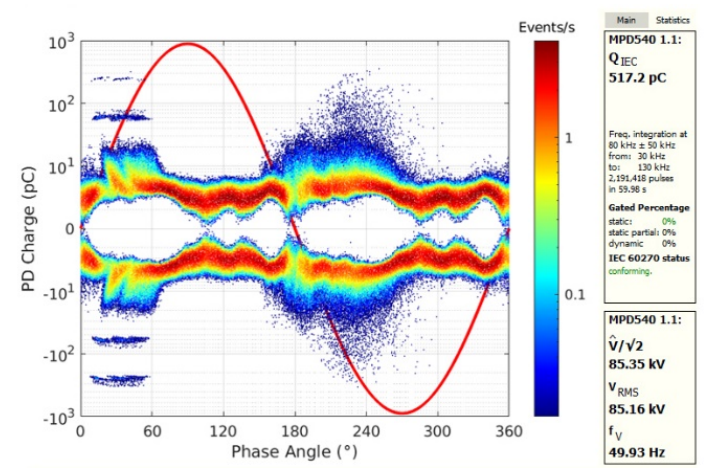

(b) Applied voltage: $75 \mathrm{kV} \mathrm{rms}$

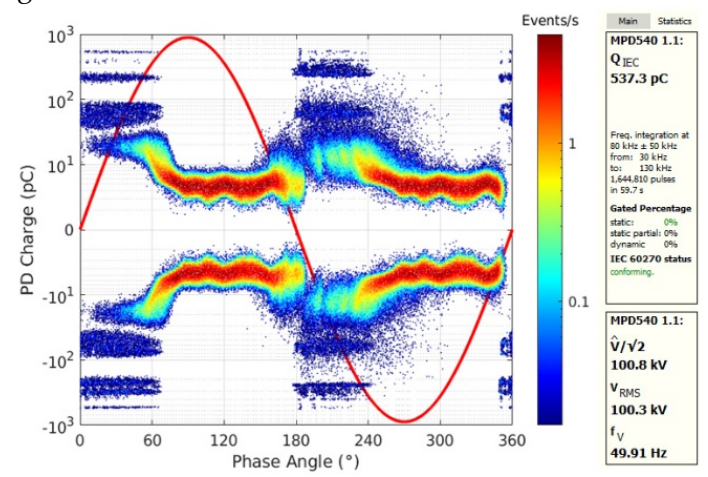

(c) Applied voltage: $100 \mathrm{kV} \mathrm{rms}$

Figure 8. PD measurement of the gas insulated line demonstrator filled with 3 bar $\mathrm{CO}_{2}$-particle-contaminated system-measured directly with a HV coupling capacitor.

\subsection{UHF Partial Discharge Measurement-Clean and Particle-Contaminated System}

During experimentation with the gas insulated line, measuring partial discharge with a wide band measurement system coupled with the HV arm of a divider as the coupling capacitor directly to the transformer, significant effects of PD external to the test GIL were observed. Therefore, it was decided to utilise the UHF sensor signals in order to mitigate some of the effects arising from external PD as seen in Figures 6-8. In order to obtain UHF measurements, a capacitive plate sensor was placed within the enclosure of the gas insulated line. This was then attached to a device which lowers the 
bandwidth and allows for direct recording from the higher frequency UHF sensor at the same time as the HV arm of a divider with coupled capacitive measurements, as shown in Figure 8. UHF data from the gas insulated line demonstrator as a clean system filled with 3 bar $\mathrm{CO}_{2}$ with an applied voltage of $100 \mathrm{kV}$ is shown in Figure 9a.

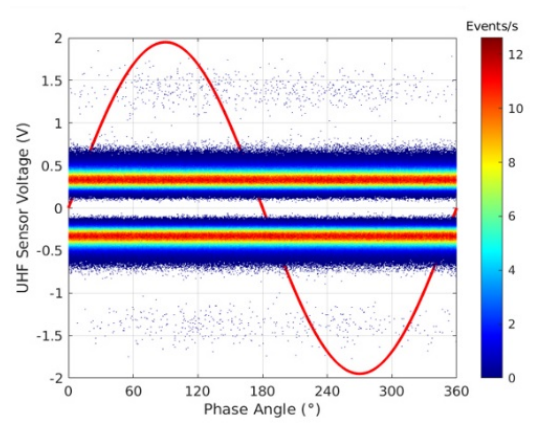

(a) Clean system at applied voltage $100 \mathrm{kV}$ rms

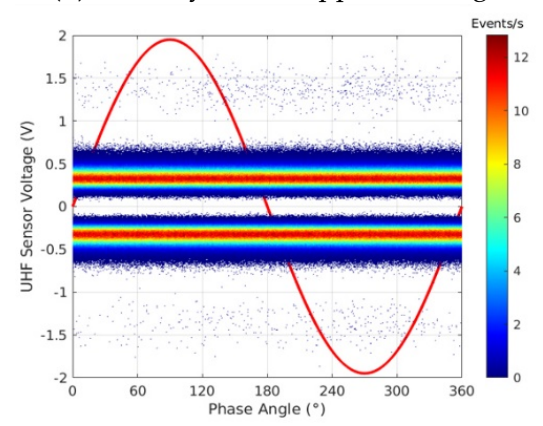

(c) Particle-contaminated system at $75 \mathrm{kV}$ rms
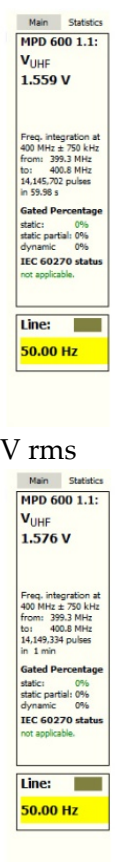

Figure 9. PD measurement of a gas insulated line demonstrator filled with 3 bar $\mathrm{CO}_{2}$ measured using a capacitively coupled UHF sensor.

When the results of the UHF measurements for the clean system, shown in Figure 9a, are compared with the results of the contaminated system in Figure 9 (b) $50 \mathrm{kV}$, (c) $75 \mathrm{kV}$ and (d) $100 \mathrm{kV}$, it can be seen that the measured PD with the presence of metallic particles is visible between the system background noise and the PD level shown above 1.25 V. In Figure 9d, the effect of the PD caused by the metal particles is the most visible when compared to Figure 9a, which is the clean system at the same $100 \mathrm{kV}$ applied voltage. The particle-contaminated system shows much more recorded PD just above the background noise of the system and before $1.25 \mathrm{~V}$, whereas the clean system PD recordings have a clear gap between the background PD and the PD above $1.25 \mathrm{~V}$. This repetitive pattern is not consistent with those of free conducting particles in gas insulated equipment where a particle becomes charged by induction under the influence of the power-frequency electric field, this particle then lifts off as the induced charge overcomes the particles weight. Eventually, a free floating particle discharges as charge is exchanged between the particle and the enclosure. Following this, the particle falls and bounces on the enclosure and the sequence may repeat [18]. At the peak of the AC wave, the electric field is highest, so free particles will gain the highest charge which proportional to the value of voltage at that point and so the PD pattern shape will form an envelope inside the AC wave, as shown in [19]. The observed PD patterns shown in Figure 9 are consistent with the expected behaviour of free metallic particles in $\mathrm{SF}_{6}$ insulated GIL free motion, where particles bounce on the outer enclosure of a section [20]. Bouncing particles on the enclosure do not form an AC wave envelope as a particle does during liftoff but rather PD across all phase angles, which is similar to those seen in the findings in this paper. This would be considered a more severe discharge than particles at a pre-liftoff stage of motion, where PD amplitudes tend to be enveloped by the shape of the AC reference wave. This also indicates that the particles measured in Figure $9 \mathrm{~d}$ are bouncing on the inner enclosure of the gas insulated line demonstrator at an 
applied voltage of $100 \mathrm{kV}$ rms but do not present a deleterious threat to the insulation system at these voltages unless they become trapped in the spacer-chamber junction. The noise immunity and density of UHF measurements are evident in the system's ability to detect and identify these low-amplitude discharges despite external effects on the connecting equipment.

\section{Discussion and Conclusions}

This research demonstrates the use of pure $\mathrm{CO}_{2}$ as an insulation medium in high voltage gas insulated equipment including transmission lines and switchgear, and it could also potentially be useful in benchmarking the PD characteristics and phase-resolved partial discharge (PRPD) patterns of future gas insulation mixtures that are currently being trialed to replace $\mathrm{SF}_{6}$, as many of these consist of high ratios of $\mathrm{CO}_{2}$. The experimentation undertaken shows the effectiveness of pure $\mathrm{CO}_{2}$ to insulate high voltage equipment, previously designed for use with $\mathrm{SF}_{6}$, up to a voltage of $242 \mathrm{kV} \mathrm{rms}$. However, it is possible to conclude that this system likely has more partial discharge than the same system insulated with $\mathrm{SF}_{6}$ at the same pressure. It is also likely that increasing the total filling pressure of $\mathrm{CO}_{2}$ may improve the system's PD performance when compared to the results presented in this paper, including its use at voltages greater than $242 \mathrm{kV}$ rms. It was demonstrated that a capacitively coupled PD measurement through a divider's HV capacitors can show bouncing particles in gas insulated equipment as well as UHF. The results confirm the usefulness of an internal UHF sensor to mitigate external noise effects from system PD, demonstrating that it is an effective tool for continued use in this type of application. Future research will determine the PD system response of other natural atmospheric gases filled in conventional high voltage gas insulated equipment including increased pressure ratings.

Author Contributions: Conceptualization, P.W. and A.H.; methodology, P.W., C.S. and A.R; software, D.C., P.W. and A.R; validation, P.W., C.S., D.C. and A.R.; formal analysis, P.W., D.C. and A.R; investigation, P.W., D.C. and M.H.; resources, P.W. and A.H.; data curation, P.W. and D.C.; writing-original draft preparation, P.W.; writing - review and editing, P.W., A.R. and D.C.; visualization, P.W.; supervision, A.H.; funding acquisition, A.H.. All authors have read and agreed to the published version of the manuscript.

Funding: This research was funded by the European Union and the Welsh European Regional Development Fund, Flexible Integrated Energy Systems (FLEXIS), (http://www.flexis.wales/) and the EPSRC Top \& Tail Demonstrator Project entitled 'Design, Construction and Characterisation of a full-scale $400 \mathrm{kV}, \mathrm{CF}_{3} \mathrm{I}$ Gas Insulated Line Demonstrator', EP/I031707/1, with supporting industrial partner National Grid.

Acknowledgments: The authors would like to thank National Grid for their support and use of the gas insulated equipment used throughout the practical testing in this paper.

Conflicts of Interest: The authors declare no conflict of interest.

\section{References}

1. Widger, P.; Haddad, A. Evaluation of SF6 leakage from gas insulated equipment on electricity networks in Great Britain. Energies 2018, 11, 2037. [CrossRef]

2. Myhre, G.; Shindell, D.; Bréon, F.M.; Collins, W.; Fuglestvedt, J.; Jianping, H.; Koch, D.; Lamarque, J.F.; David, L.; Mendoza, B.; et al. Anthropogenic and Natural Radiative Forcing; Cambridge University Press: Cambridge, UK, 2013.

3. Intergovernmental Panel on Climate Change (IPCC). Working Group I Contribution to Fourth Assessment Report of the Intergovernmental Panel on Climate Change; Cambridge University Press: Cambridge, UK, 2007.

4. Li, Y.; Zhang, X.; Zhang, J.; Xiao, S.; Xie, B.; Chen, D.; Gao, Y.; Tang, J. Assessment on the toxicity and application risk of C4F7N: A new SF6 alternative gas. J. Hazard. Mater. 2019, 368, 653-660. [CrossRef] [PubMed]

5. Beroual, A.; Haddad, A. Recent Advances in the Quest for a New Insulation Gas with a Low Impact on the Environment to Replace Sulphur Hexafluoride (SF6) Gas in High-Voltage Power Network Applications. Energies 2017, 10, 1216. [CrossRef]

6. Rabie, M.; Franck, C.M. Assessment of Eco-friendly Gases for Electrical Insulation to Replace the Most Potent Industrial Greenhouse Gas SF6. Environ. Sci. Technol. 2018, 52, 369-380. [CrossRef] [PubMed] 
7. Zhang, X.; Li, Y.; Xiao, S.; Tang, J.; Tian, S.; Deng, Z. Decomposition Mechanism of C5F10O: An Environmentally Friendly Insulation Medium. Environ. Sci. Technol. 2017, 51, 10127-10136. [CrossRef] [PubMed]

8. Widger, P.; Haddad, A.; Griffiths, H. Breakdown performance of vacuum circuit breakers using alternative CF3I-CO2 insulation gas mixture. IEEE Trans. Dielectr. Electr. Insul. 2016, 23, 14-21. [CrossRef]

9. Widger, P.; Griffiths, H.; Haddad, A. Insulation strength of CF3I-CO2 gas mixtures as an alternative to SF6 in MV switch disconnectors. IEEE Trans. Dielectr. Electr. Insul. 2018, 25, 330-338. [CrossRef]

10. Seeger, M.; Avaheden, J.; Pancheshnyi, S.; Votteler, T. Streamer parameters and breakdown in CO2. IOP J. Phys. D Appl. Phys. 2016, 50, 015207. [CrossRef]

11. Ohtsuka, S.; Onomoto, M.; Nishimura, T.; Matsumoto, S.; Shibuya, M.; Hikita, M. Insulation Properties of High-pressure CO2 Gas Under Existence of a Particle. In Proceedings of the European Power and Energy Systems (EuroPES 2003) Conference, Marbella, Spain, 3-5 September 2003; ACTA Press: Calgary, AB, Canada, 2003.

12. Widger, P.; Haddad, A. Analysis of Gaseous By-Products of CF3I and CF3I-CO2 after High Voltage Arcing Using a GCMS. Molecules 2019, 24, 1599. [CrossRef] [PubMed]

13. High-Voltage Switchgear and Controlgear-Part 203: Gas-Insulated Metal-Enclosed Switchgear for Rated Voltages above 52 kV, IEC/British Standard BS62271-203, 2012. Available online: https://shop.bsigroup.com/ ProductDetail?pid=000000000030179469 (accessed on 1 March 2020).

14. High-Voltage Switchgear and Controlgear-Part 204: Rigid Gas-Insulated Transmission Lines for Rated Voltage above 52 kV, IEC/British Standard BS62271-204, 2011. Available online: https://shop.bsigroup.com/ ProductDetail?pid=000000000030205411 (accessed on 3 March 2020).

15. High-Voltage Test Techniques-Part 60270: Partial Discharge Measurements, IEC/British Standard BS60270, 2001, +A1, 2016. Available online: https://shop.bsigroup.com/ProductDetail/?pid=000000000030228771 (accessed on 12 December 2019).

16. BOC, Carbon Dioxide Materials Safety Data Sheet. 2017. Available online: https://www.boconline.co.uk/en/ legacy/attachment?files=tcm:4410-39607,tcm:410-39607,tcm:10-39607 (accessed on 5 January 2020).

17. Simply Bearings Ltd, 316 Stainless Steel Data Sheet. 2020. Available online: https://simplybearings.co.uk/ shop/files/316.pdf (accessed on 10 January 2020).

18. White, A.; Coventry, P.; Jones, G.R. Condition monitoring of high voltage equipment. In High Voltage Engineering and Testing, 2nd ed.; Ryan, H.M., Ed.; The Institution of Electrical Engineers: London, UK, 2001; pp. 642-643.

19. Reid, A.J.; Judd, M.D.; Fouracre, R.A.; Stewart, B.G.; Hepburn, D.M. Simultaneous Measurement of Partial Discharges Using IEC60270 and Radio Frequency Techniques. IEEE Trans. Dielectr. Electr. Insul. 2011, 18, 444-455. [CrossRef]

20. Irwin, T.; Lopez-Roldan, J.; Charlson, C. Partial Discharge Detection of Free Moving Particles in GIS by the UHF Method: Recognition Pattern Depending on the Particle Movement and Location. In Proceedings of the IEEE Power Engineering Society Winter Meeting (Cat. No.00CH37077), Singapore, 23-27 January 2000; ISBN 0-7803-5935-6. [CrossRef]

(C) 2020 by the authors. Licensee MDPI, Basel, Switzerland. This article is an open access article distributed under the terms and conditions of the Creative Commons Attribution (CC BY) license (http://creativecommons.org/licenses/by/4.0/). 